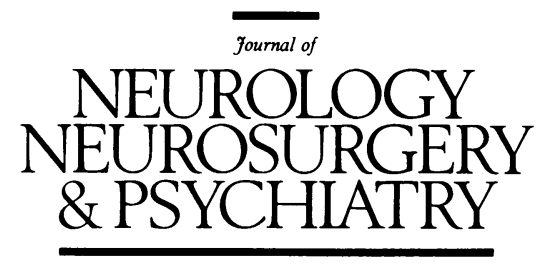

Editorial

\title{
Non Alzheimer's disease forms of cerebral atrophy
}

In the not too distant past Alzheimer's disease was considered sufficiently ubiquitous to permit its diagnosis in the elderly demented, once cerebrovascular disease, cerebral trauma and alcohol abuse had been excluded. An attempt to use inclusion rather than exclusion criteria for "probable" or "possible" Alzheimer's disease is contained in the Diagnostic Statistical Manual III. The latter now serves as holy writ in the search for clinical confirmation of Alzheimer's disease to the neglect of forms of cerebral atrophy which may be clinically and pathologically distinct from Alzheimer's disease and yet meet its descriptive psychiatric diagnostic criteria.

The last few years have witnessed a directional change in the investigation of primary cerebral atrophy with qualitative neuropsychological analysis of clinical syndromes allied to more functional brain imaging techniques and histological verification. Some new and distinct cerebral disorders have emerged, which are much more common than previously supposed and those reviewed here comprise dementia frontal-lobe type (DFT), DFT and motor neuron disease (MND), lobar atrophy and diffuse Lewy body disease.

\section{Dementia of frontal-lobe type (DFT)}

Studies from Manchester, United Kingdom ${ }^{1}$ and Lund, Sweden ${ }^{2}$ have delineated the form of non-Alzheimer's disease dementia designated as dementia of frontal-lobe type (DFT). Two further studies from these centres ${ }^{34}$ have independently confirmed that DFT, as a form of cerebral atrophy distinct from Alzheimer's disease, is more common than previously supposed. This disorder occurs at an earlier age than Alzheimer's disease and approximately half the patients have a parent with dementia. No sexual preponderance is seen, as in Alzheimer's disease, and in the British series, the ratio of the incidence of DFT to Alzheimer's disease was estimated at 1:4.

Patients present with a striking change in personality and social conduct, followed by progressive impairment of speech, with preservation of visuo-spatial abilities until the end stages of the disease. Stereotyped behaviour and hyper-oral tendencies are characteristic. Neurological signs are minimal and confined to the emergence of primitive reflexes. Formal neuropsychological testing confirms a "frontal lobe syndrome". The electroencephalogram is normal and regional cerebral blood flow is selectively reduced in the anterior cerebral hemispheres. ${ }^{5}$

Biopsy tissue examination excludes Alzheimer's disease, and necropsy ${ }^{6}$ reveals striking atrophy of the frontal and temporal lobes with loss of large cortical neurons, cortical spongiform change and both cortical and sub-cortical astrocytic gliosis. Neurofibrillary tangles and senile plaques are absent; in only four out of 20 patients with the syndrome were balloon cells and neuronal inclusion bodies, compatible with Pick's disease, observed. The precise status of DFT in relationship to Pick's disease requires clarification, but it is a disorder distinct from Alzheimer's disease on clinical, neurophysiological, pathological and demographic grounds.

\section{Dementia of frontal-lobe type and motor neuron disease}

In general patients with DFT survive for many years and some have survived for over fifteen years from onset. However, four patients have been described, ${ }^{7}$ in whom a profound and rapidly progressive dementia occurred in association with clinical features of motor neuron disease. Mental changes occurred first and the pattern of dementia indicated impaired frontal lobe function, confirmed by reduced tracer uptake in the frontal lobes on single photon emission computerised tomography (SPECT). Later weakness, wasting and fasciculations of limb muscles emerged and progressive bulbar palsy led to death within two years of onset. Electrophysiological studies confirmed widespread muscular denervation compatible with motor neuron disease.

Pathological examination of the brains of three patients revealed fronto-temporal lobe atrophy, with loss of large pyramidal neurons, mild gliosis and spongiform change. Similar changes were found in the corpus striatum and thalamus and also there was significant cell loss and gliosis in the substantia nigra. Depletion of neurons in the hypoglossal nuclei and anterior horns of the spinal cord, was not accompanied by significant changes in the cortico-bulbar and cortico-spinal tracts. The pathological findings mirror closely those of Japanese patients with dementia and motor neuron disease. ${ }^{8}$

Previous studies have failed to define the precise clinical characteristics of the associated dementia. However in this most recent study both the clinical picture and pathological findings resembled those of DFT and were distinct from those of Alzheimer's disease.

It is clear that the high familial incidence of DFT demands further cytogenetic studies to shed light on the associated motor neuron disease and other forms of primary degenerative dementia.

\section{Lobar atrophy}

Awareness of DFT and other cerebral degenerations with non-Alzheimer's pathology such as the syndrome of slowly progressive aphasia, ${ }^{910}$ ought to awaken investigators to the possibility of additional forms of focal atrophy. Slowly progressive aphasia first described by Wechsler ${ }^{14}$ consists of speech reduction progressing to mutism in the relative absence of other neuropsychological deficits. Imaging studies indicated a selective disorder of the left dominant 
cerebral hemisphere, confirmed pathologically and demonstrated to be due to a loss of large cortical neurons with spongiform change and astrocytic gliosis asymmetrically involving the dominant hemisphere.

Case reports have proliferated. ${ }^{12-16}$ However, descriptions of the language disorder vary and the extent to which other deficits emerge with disease progression appears not to be uniform. Although a minority of cases has come to necropsy, ${ }^{17-20}$ debate regarding the aetiological status of progressive aphasia remains unresolved: specifically whether the cases represent focal representations of Alzheimer's disease, ${ }^{1421}$ or Pick's disease, ${ }^{17} 1820$ or constitute a distinct disease entity. ${ }^{9}$

The relatively large number of cases of progressive aphasia in the literature, presumably reflects the vulnerability of language functions to cerebral disease and it is predicted that selective neuropsychological deficits will be associated with focal degenerations in the right cerebral hemisphere. Pathological evidence will be required to bring unity to increasing numbers of clinically based observations.

Benson et al $^{22}$ have described the clinical syndrome of "posterior cortical atrophy" which is likely to represent lobar atrophy of the parieto-occipital cortex. Five patients presented with early and severe ataxia, agraphia, acalculia, anomia, visual agnosia and disorientation, disorders of ocular fixation and a transcortical sensory aphasia. Memory, insight and personal conduct were strikingly preserved and the neurological examination was normal. Computerised tomography and magnetic resonance imaging revealed cerebral atrophy most marked in the posterior hemispheres. Pathological verification indicates nonAlzheimer's disease in some cases and in others, forms of Alzheimer's disease in which the pathological changes appear preferentially to affect the parietal occipital association cortex in the early stages of progression.

A case has been described of progressive apraxia in the absence of any other neuropsychological deficits ${ }^{23}$ and atrophy localised to the superior parietal lobules has been demonstrated on SPECT. Progressive apraxia has also been described in association with apperceptive visual agnosia. ${ }^{24}$ Unfortunately, pathological characterisation was lacking from these case descriptions.

\section{Lewy body disease}

Japanese investigators described the syndrome of dementia and rigidity in which Lewy bodies occurred in mid-line nuclei with or without cortical neuro-fibrillary tangles and senile plaques. ${ }^{25} 26$ Unfortunately, clinical psychological descriptions were meagre. Interest in "Lewy body disease" was kindled by a description of patients initially thought to have Parkinson's disease because of akinesia and rigidity but who had striking and early fluctuating mental changes of a sub-acute confusional state with behavioural disorder and hallucinations. Necropsy revealed Lewy bodies not only in the substantia nigra but widespread throughout the cerebral cortex. ${ }^{27}$

The Nottingham group went on to demonstrate the superiority of anti-ubiquitin immunocytochemistry over conventional techniques in the detection of diffuse Lewy bodies. ${ }^{28}$ Utilising the technique, they described the clinical and pathological features of fifteen cases of diffuse Lewy body disease. ${ }^{29}{ }^{30}$ Fifteen brains with the pathological hallmarks of diffuse Lewy body disease had been collected in one centre in one year. The patients had been longitudinally studied, neurologically and psychologically with sufficient cognitive measures to permit the rating of severity of dementia on a five point scale. Of equal incidence were patients who had presented with Parkin- son's disease and later developed dementia, and patients who had presented with dementia and who later developed Parkinson's disease. The form of Parkinson's disease was indistinguishable from idiopathic Pàrkinson's disease and the motor symptoms were responsive to levodopa. The dementia was "cortical" in type with amnesia, aphasia, visuo-spatial disorientation and apraxia running a fluctuating course and complicated by hallucinations, agitation and confusion.

Pathologically, Lewy bodies were identified profusely in the cerebral cortex and their numbers were highly correlated with the severity of dementia. There was neuronal loss in the substantia nigra with Lewy bodies in the surviving neurons, but there was no correlation with these sub-cortical changes and the severity of dementia. This was true for similar changes in the nucleus basalis. Of considerable interest was the presence of large numbers of cortical senile plaques, the density of which correlated with that of cortical Lewy bodies. Only a few brains were found to contain neurofibrillary tangles and their numbers did not correlate with those of senile plaques or the severity of dementia. Similar clinical pathological findings have been documented by Perry et $a l,{ }^{31}$ who have chosen the title "Senile Dementia of Lewy body type", to designate this group of patients, thought to rank second in frequency to Alzheimer's disease as the cause of primary cerebral atrophy in the elderly. Previously the patients had been clinically diagnosed as forms of vascular encephalopathy because of the fluctuating clinical course.

It is suggested that Parkinson's disease and diffuse Lewy body disease represent clinical syndromes determined by the distribution of Lewy bodies in the brain stem and cerebral cortex. Senile plaques must be seen as non-specific changes which do not necessarily denote the presence of Alzheimer's disease. This view is supported by the studies of Gibb et $a l,^{32}$ where again the clinical syndrome of Parkinson's disease and "cortical dementia" was associated with a distribution of Lewy bodies in the brain stem and cerebral cortex and significant changes of Alzheimer's disease were absent.

It is evident from a survey of recent investigations into dementia that not all primary cerebral atrophy represents Alzheimer's disease and it is likely that fresh clinical pathological designations will proliferate. Such progress will be made by adopting neuropsychological analytical techniques whith specifiy the qualitative nature of clinical syndromes rather than submerging differences under a weight of numbers generated by standard clinical psychological battery tests. The quantification and rating of the severity of dementia should follow the specification of the dementia syndrome. Distinct neurological syndromes can now be evaluated in life using functional imaging such as SPECT and PET which are capable of generating topographical cerebral correlates of behaviour. The pathological evaluation of brains, using morphometric histological analyses and the new dynamic techniques is bound to be most informative when correlated with quantitative neuropsychological measures made during life.

Principally, conceptual progress is likely to be furthered by rejecting the notion that dementia represents a nonspecific breakdown in intellect and memory, rather than distinct neuropsychological syndromes characterising particular cerebral disorders and their pathological and functional differentiation within the brain.

D NEARY

Department of Neurology,

Manchester Royal Infirmary, Manchester 
1 Neary D, Snowden JS, Bowen DM, et al. Neuro-psychological syndromes in presenile dementia due to cerebral atrophy. J Neurol Neurosurg Psychiatry presenile dementia

2 Gustafson L, Brun A, Frank Holmvist A, Risberg J. Regional cerebral blood flow in degenerative frontal lobe dementia of non-Alzheimer's type. $J$ Cereb Blood Flow Metab 1985;5(suppl 1):141-2.

3 Gustafson L. Frontal lobe degeneration of non-Alzheimer type. II. Clinical picture and differential diagnosis. Arch Gerontol Geriatr 1987;6:209-23.

4 Neary D, Snowden JS, Northen B, et al. Dementia of frontal lobe type. J Neurol Neurosurg Psychiatry 1988;51:353-61.

5 Risberg J. Frontal lobe degeneration of non-Alzheimer type. III. Regional cerebral blood flow. Arch Gerontol Geriatr 1987;6:225-33.

6 Brun A. Frontal lobe degeneration of non-Alzheimer type. I. Neuropathology. Arch Gerontol Geriatr 1987;6:193-208.

7 Neary D, Snowden JS, Mann DMA, et al. Frontal lobe dementia and motor neuron disease. J Neurol Neurosurg Psychiatry 1990;53:23-32.

8 Morita K, Kaiya H, Ikeda T, Namba M. Presenile dementia combined with amyotrophy: a review of 34 Japanese cases. Arch Gerontol Geriat 1987;6:263-77.

9 Mesulam MM. Slowly progressive aphasia without generalised dementia. Ann Neurol 1982;11:592-8.

10 Kirshner HS, Webb WG, Kelly MP, Wells CE. Language disturbance-an initial symptom of cortical degenerations and dementia. Arch Neurol 1984;41:491-6.

11 Wechsler AF. Presenile dementia presenting as aphasia. J Neurol Neurosurg Psychiatry 1977;40:303-5.

12 Heath PD, Kennedy P, Kapur N. Slowly progressive aphasia without generalised dementia. Ann Neurol 1983;13:687-8.

13 Chawluk JB, Mesulam MM, Hurtig H, et al. Slowly progressive aphasia without generalised dementia: studies with positron emission tomograwithout generalised dementia: stud

14 Poeck K, Luzzatti C. Slowly progressive aphasia in three patients. Brain 1988;111:151-68.

15 Goulding PJ, Northen B, Snowden JS, et al. Progressive aphasia with right sided extrapyramidal signs: another manifestation of localised cerebral atrophy. J Neurol Neurosurg Psychiatry 1989;52:128-30.

16 Snowden JS, Goulding PJ, Neary D. Semantic dementia: a form of circumscribed cerebral atrophy. Behav Neurol 1989;2:167-82.

17 Wechsler AF, Verity A, Rosenschein S, et al. Pick's disease. A clinical computed tomographic and histologic study with golgi impregnation observations. Arch Neurol 1982;39:287-90.

18 Holland AL McBurney DH, Moossy J, Reinmuth OM. The dissolution of language in Pick's disease with neurofibrillary tangles: a case study. Brain Lang 1985;24:36-58.

19 Kirshner HS, Tanridag O, Thurman L, Whetsell WO. Progressive aphasia without dementia: two cases with focal spongiform degeneration. Ann Neurol 1987;22:527-32.

20 Graff-Radford NR, Damasio AR, Hyman BT, et al. Progressive aphasia in a patient with Pick's disease: a neuropsychological, radiologic and anatomic study. Neurology 1990;40:620-6.

21 Green J, Morris JC, Sandson J, et al. Progressive aphasia a precursor of global dementia? Neurology 1990;40:423-9.

22 Benson DF, Davis RJ, Snyder BD. Posterior cortical atrophy. Arch Neurol 1988;45:789-93.

23 Dick JPR, Snowden JS, Northen B, et al. Slowly Progressive Apraxia. Behavioural Neurology 1989;2:101-14.

24 De Renzi E. Slowly progressive visual agnosia or apraxia without dementia. Cortex 1986;22:171-80.

25 Kosaka K. Lewy bodies in cerebral cortex. Report of 3 cases. Acta Neuropathol 1978;42:127-34.

26 Kosaka K, Tsuchiya K, Yoshimura M. Lewy body disease with and without dementia: a clinicopathological study of 35 cases. Clin Neuropathol 1988;7:299-305.

27 Byrne EJ, Lowe J, Godwin-Austen RB. Dementia and Parkinson's disease associated with diffuse cortical Lewy bodies. Lancet 1987;i:501.

28 Lennox G, Lowe J, Morrell K, et al. Anti-ubiquitin immunocytochemistry is more sensitive than conventional techniques in the detection of diffuse Lewy body disease. J Neurol Neurosurg Psychiatry 1989;52:67-71.

29 Byrne EJ, Lennox G, Lowe J, et al. Diffuse Lewy body disease: clinical features in fifteen cases. $J$ Neurol Neurosurg Psychiatry 1989;52:709-17.

30 Lennox G, Lowe J, Landon M, et al. Diffuse Lewy body disease: correlative neuropathology using anti-ubiquitin immunocytochemistry. $J$ Neurol neuropathology using anti-ubiquitin
Neurosurg Psychiatry 1989;52:1236-47.

31 Perry RH, Irving D, Blessed G, et al. Clinically and neuropathologically distinct form of dementia in the elderly. Lancet 1989;i:166.

32 Gibb WRG, Luthert PJ, Janota I, et al. Cortical Lewy body dementia clinical features and classification. J Neurol Neurosurg Psychiatry 1989;52:185-92. 